EPJ Web of Conferences 47, 05005 (2013)

DOI: $10.1051 /$ epjconf/20134705005

(C) Owned by the authors, published by EDP Sciences, 2013

\title{
Jupiter analogues and planets of active stars
}

\author{
M. Kürster ${ }^{1, a}$, M. Zechmeister ${ }^{2}$, M. Endl ${ }^{3}$, G. Lo Curto ${ }^{4}$, H. Hartman ${ }^{5,6}$, \\ H. Nilsson ${ }^{6}$, T. Henning ${ }^{1}$, A.P. Hatzes ${ }^{7}$ and W.D. Cochran ${ }^{3}$
}

\author{
${ }^{1}$ Max-Planck-Institut für Astronomie, Königstuhl 17, 69117 Heidelberg, Germany \\ ${ }^{2}$ Institut für Astrophysik, Georg-August-Universität, Friedrich-Hund-Platz 1, 37077 Göttingen, \\ Germany \\ ${ }^{3}$ McDonald Observatory, University of Texas, Austin, TX78712, USA \\ ${ }^{4}$ European Southern Observatory, Karl-Schwarzschild-Str. 2, 85748 Garching, Germany \\ ${ }^{5}$ Applied Science, School of Technology, Malmö University, Sweden \\ ${ }^{6}$ Lund Observatory, Lund University, Box 43, 22100 Lund, Sweden \\ ${ }^{7}$ Thüringer Landessternwarte Tautenburg, Sternwarte 5, 07778 Tautenburg, Germany
}

\begin{abstract}
Combined results are now available from a 15 year long search for Jupiter analogues around solar-type stars using the ESO CAT + CES, ESO $3.6 \mathrm{~m}+\mathrm{CES}$, and ESO $3.6 \mathrm{~m}+$ HARPS instruments. They comprise planet (co-)discoveries ( $l$ Hor and HR 506) and confirmations (three planets in HR 3259) as well as non-confirmations of planets (HR 4523 and $\epsilon$ Eri) announced elsewhere. A long-term trend in $\epsilon$ Ind found by our survey is probably attributable to a Jovian planet with a period $>30 \mathrm{yr}$, but we cannot fully exclude stellar activity effects as the cause. A 3.8 year periodic variation in HR 8323 can be attributed to stellar activity.
\end{abstract}

\section{INTRODUCTION}

In 1992 a readial velocity (RV) search program for extrasolar planets around solar-type stars was begun (Kürster et al. 2000; Endl et al. 2002) with the ESO $1.4 \mathrm{~m}$ CAT telescope and the Coudé Echelle spectrograph (CES) together with its Long Camera (LC) which provided a resolving power $R=100,000$. In 1999 the Long Camera was replaced by the $R=230,000$ Very Long Camers (VLC) fibre fed to the ESO $3.6 \mathrm{~m}$ telescope with which the survey was continued up to the time when some temporal overlap with the higher precision ESO HARPS spectrograph $(R=130,000$, wide wavelength coverage, also at the ESO $3.6 \mathrm{~m}$ telescope) could be attained which was finally employed for the survey from 2003-2007. Archival HARPS data from other programs were added extending our time baseline into 2009 for a few stars.

A total of 31 bright $(V<6)$ solar-type stars was monitored throughout the whole 15-year survey. The main goal of the long-term studies of this sample was the search for Jupiter analogues. Despite the fact that the stars in the sample were selected for low activity levels, the more active stars turned out to be the most interesting targets. This contribution summarizes the most important results. The survey and its results for the whole sample are described in much more detail in Zechmeister et al. (2013).

\footnotetext{
ae-mail: kuerster@mpia.de
}

This is an Open Access article distributed under the terms of the Creative Commons Attribution License 2.0, which permits unrestricted use, distribution, and reproduction in any medium, provided the original work is properly cited. 


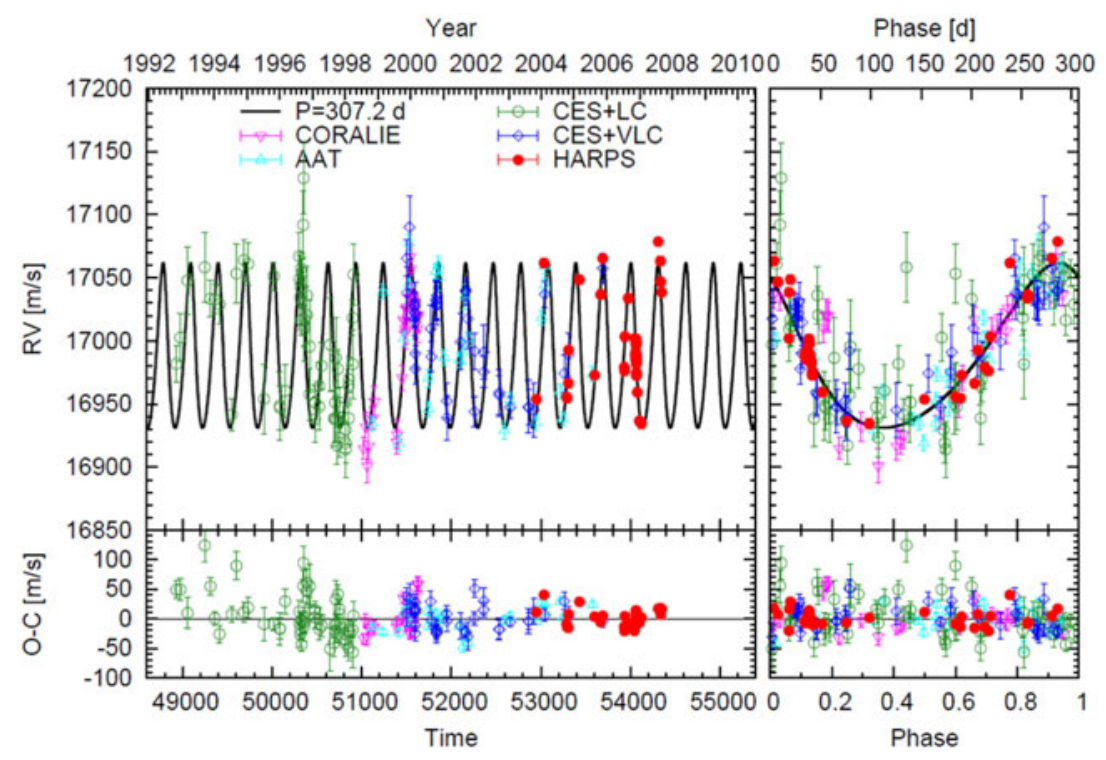

Figure 1. Left: RV time series for $\imath$ Hor combined with data from the Anglo-Australian Telescope (AAT; Butler et al. 2001) and from the $1.2 \mathrm{~m}$ EULER-Telescope + CORALIE spectrograph (Naef et al. 2001). Right: RVs phase folded with the orbital period of $P=307 \mathrm{~d}$. Bottom panels show the residuals from the Keplerian fit.

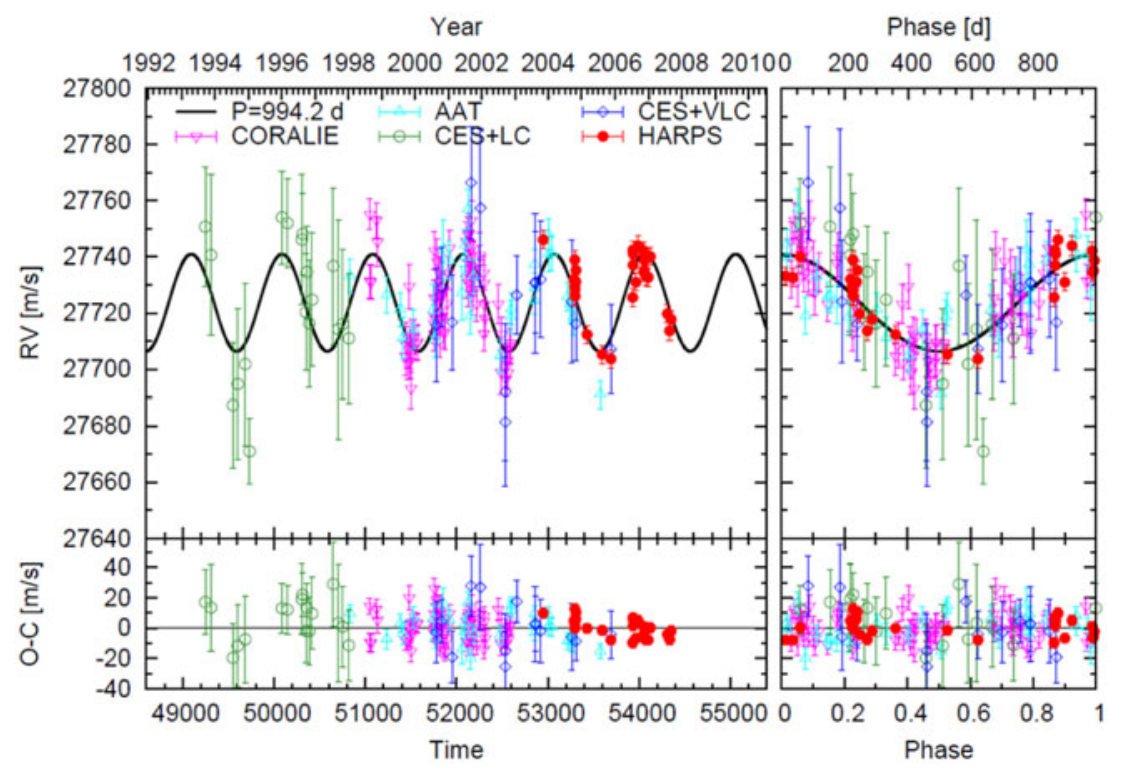

Figure 2. Left: RV time series for HR 506 combined with AAT data (Butler et al. 2006) and CORALIE data (see http://obswww . unige.ch/ udry/planet/hd10647. html). Right: RVs phase folded with the orbital period of $P=995 \mathrm{~d}$. Bottom panels show the residuals from the Keplerian fit.

\section{RESULTS}

- (Co-)discoveries. Fig. 1 shows the RV time series for the G0V active star $\imath$ Hor revealing a planetary companion with a minimum mass of $m \sin i=2.48 \mathrm{M}_{\mathrm{Jup}}$ and an orbital period of $P=307.2 \mathrm{~d}$. 


\section{Hot Planets and Cool Stars}

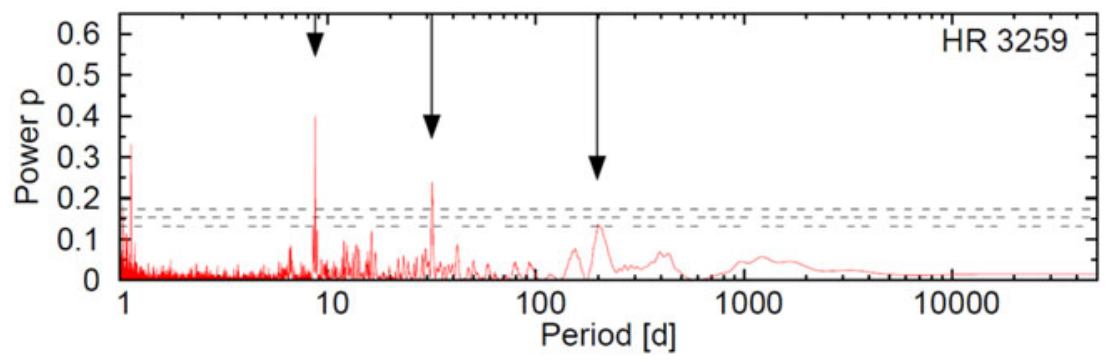

Figure 3. Generalized Lomb-Scargle (GLS) periodogram for HR 3259. The vertical arrows indicate the periods for the three planets announced by Lovis et al. (2006). See Zechmeister \& Kürster (2009) for details of the GLS.

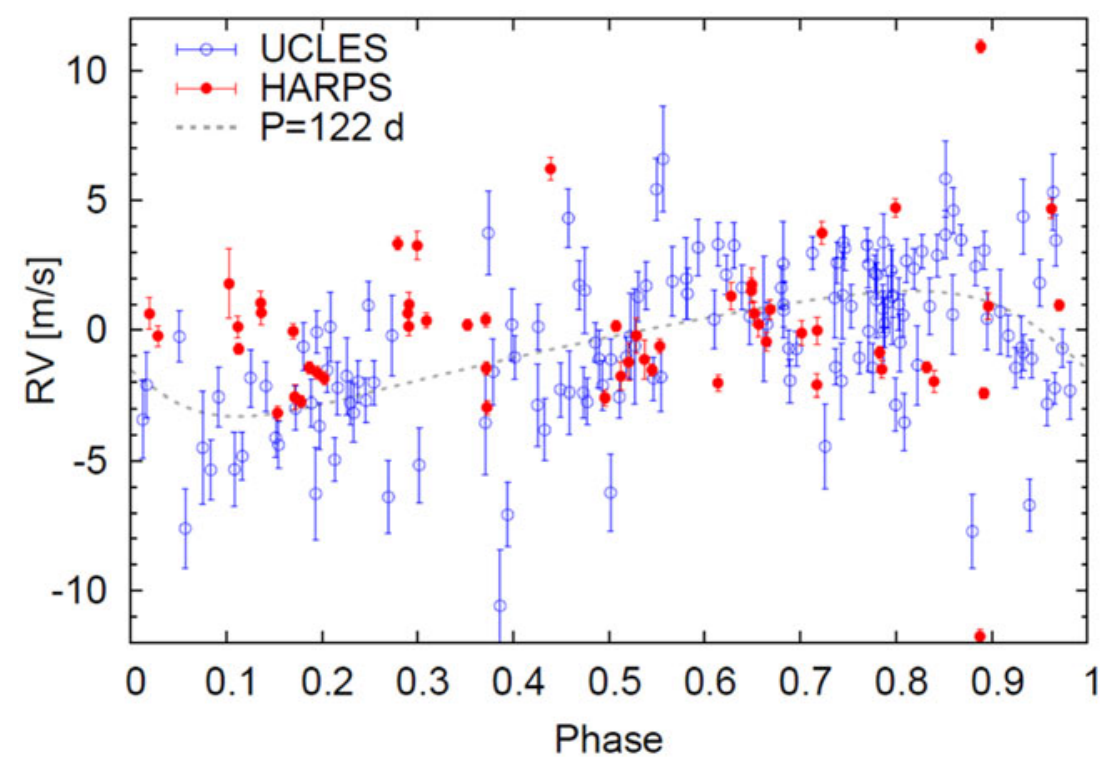

Figure 4. RV data for HR 4523 phase folded with a period of $122.1 \mathrm{~d}$. The dashed curve is an orbital solution proposed by Tinney et al. (2011) based on the UCLES data. HARPS RV data are shown for comparison. The do not support the planetary signal.

This companion which orbits on a slightly eccentric $(e=0.18)$ orbit within the habitable zone of its host star is a genuine discovery of this survey, originally announced by Kürster et al. (2000) based on the CES + LC data alone. Since it has been followed-up by different teams and with various different instruments it is among the best confirmed extrasolar planets.

RV data for the F9V star HR 506 are shown in Fig. 2 indicating a planet on a circular orbit with a period of $P=994.2 \mathrm{~d}$ and a minimum mass of $m \sin i=0.98 \mathrm{M}_{\mathrm{Jup}}$. This planet was originally announced by the Swiss team at the XIX-th IAP Colloquium in Paris (2003), but never published.

- Confirmations. Lovis et al. (2006) announced three Neptune-type planets around the G7.5V star HR 3259 with minimum masses of $10.2,11.8$, and $18.1 \mathrm{M}_{\odot}$ and periods of 8.67, 31.6, and $197 \mathrm{~d}$, respectively. We can confirm these planets with the data from our survey (mostly with the HARPS data) finding an RV signal at each of these period values, see Fig. 3.

- Non-confirmations. For the G3V star HR 4523 Tinney et al. (2011) announced a Neptune-like planet with a minimum mass of $16 \mathrm{M}_{\odot}$ and a period of $122.1 \mathrm{~d}$. In Fig. 4 we show their data phase folded with 


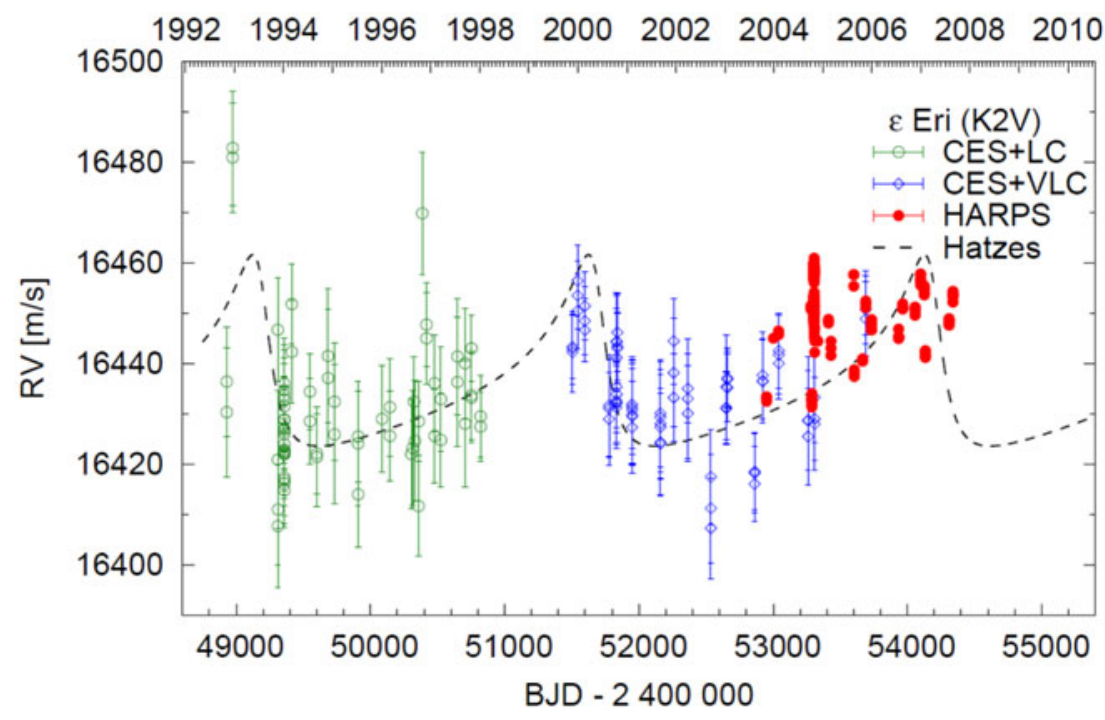

Figure 5. RV time series for $\epsilon$ Eri. The Keplerian orbit taken from Hatzes et al. (2000) is not supported by our data.

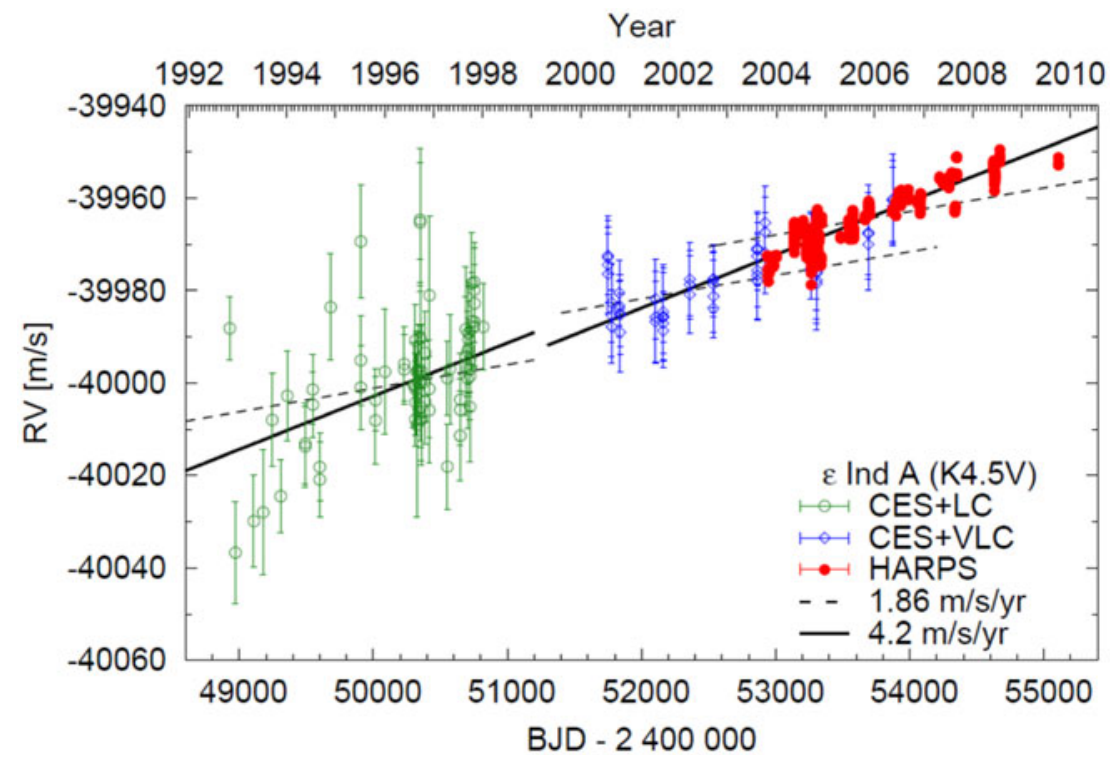

Figure 6. RV time series for $\epsilon$ Ind. Model curves are shown for a constant RV (i.e. considering only the secular acceleration of $1.86 \mathrm{~ms}^{-1} \mathrm{yr}^{-1}$; dashed line) as well as for a linear trend (solid line). The offset in the data (and the fit) occuring around BJD 2,451,200 is of instrumental nature.

this period and also our HARPS data in which we do not find any evidence for the signal. Therefore, we cannot confirm this planet.

Fig. 5 shows another case in which we cannot confirm a planet announced earlier. It provides our RV time series for the K2V star $\epsilon$ Eri with the orbit by Hatzes et al. (2000) overplotted. This orbit corresponds to a planet with a minimum mass of $0.86 \mathrm{M}_{\mathrm{Jup}}$, a period of $6.9 \mathrm{yr}$, and an eccentricity of 0.6. As the comparison shows, our data alone do not provide unequivocal evidence for the planet. 


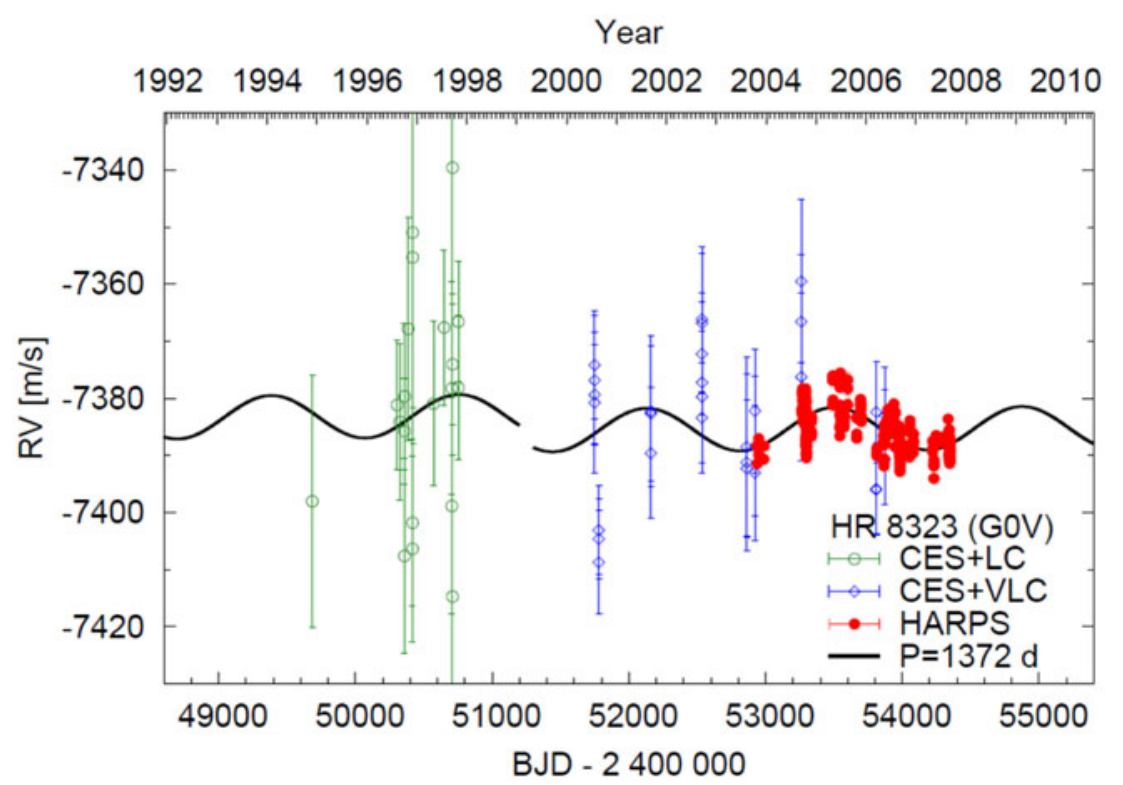

Figure 7. RV time series for HR 8323 together with a sinusoidal model. As in Fig. 6, the offset occuring around BJD 2,451,200 is of instrumental nature.

- Companions vs. stellar activity. Fig. 6 shows our RV time series for the K4V star $\epsilon$ Ind along with a linear fit. As shown in the figure, this trend exceeds the expected RV increase due to the $\mathrm{RV}$ secular acceleration which is quite substantial for this rapidly moving nearby star. The trend is likely to be produced by a long-period ( $P>30 \mathrm{yr}$ ) sub-stellar $\left(m \sin i>0.97 \mathrm{M}_{\mathrm{Jup}}\right.$ ) companion. The HARPS data make it possible to correlate the RV measurements with activity indicators, i.e. the index for chromospheric CaII emission $\log R_{\mathrm{HK}}^{\prime}$, or the FWHM and the bisector (BIS) of the average photospheric line profile (the profile of the cross-correlation with a mask employed to determine the RV). If activity is the cause for a (spurious) RV signal, then a correlation of the RV data with $\log R_{\mathrm{HK}}^{\prime}$ and also with FWHM and BIS is expected. For $\epsilon$ Ind such a correlation exists for $\log R_{\mathrm{HK}}^{\prime}$ and FWHM, but not for BIS which could indicate that the star also has a long-term trend in its activity level, but its correlation with the RV data is just a coincidence.

This is different for the G0V star HR 8323 whose RV time series is shown in Fig. 7. We find a sinusoidal variation with a period of $3.8 \mathrm{yr}$, but in this case all three activity indicators (including BIS) are correlated with the RVs so that we exclude a companion. Most likely, the periodic variation is due to a stellar activity cycle.

\section{CONCLUSION}

While our long-term RV survey of 31 solar-type stars has not found a genuine Jupiter analogue at $\approx 5 \mathrm{AU}$ separation from its host star, we have contributed discoveries of closer-in Jupiter-type planets and a possible substellar object at wide separation as well as confirmations and non-confirmations of planets found elsewhere. We have addressed the influence of stellar activity on RV signals and how it affects their interpretation. 


\section{References}

[1] Butler, R.P., Tinney, C.G., Marcy, G W., et al., ApJ, 555, 410 (2001)

[2] Butler, R.P., Wright, J.T., Marcy, G.W., et al., ApJ, 646, 505 (2006)

[3] Endl, M., Kürster, M., Els, S., et al., A\&A, 392, 671 (2002)

[4] Hatzes, A.P., Cochran, W.D., McArthur, B., et al., ApJ, 544, L145 (2000)

[5] Jones, H.R.A., Butler, R.P., Tinney, C.G., et al., in Astronomical Society of the Pacific Conference Series, 321, Extrasolar Planets: Today and Tomorrow, eds. J. Beaulieu, A. Lecavelier Des Etangs, \& C. Terquem, 298 (2004)

[6] Kürster, M., Endl, M., Els, S., et al., A\&A, 353, L33 (2000)

[7] Lovis, C., Mayor, M., Pepe, F., et al., Nature, 441, 305 (2006)

[8] Naef, D., Mayor, M., Pepe, M., et al., A\&A, 375, 205 (2001)

[9] Tinney, C.G., Butler, R.P., Jones, H.R.A., et al., ApJ, 727, 103 (2011)

[10] Zechmeister M. \& Kürster M., A\&A, 496, 577

[11] Zechmeister M., Kürster M., Endl, et al., A\&A, in press (2013); arXiv:1211.7263 (2012) 